\section{Smegmatis meets tuberculosis}

\section{By Kai-Jye Lou, Staff Writer}

Researchers at the Albert Einstein College of Medicine of Yeshiva University have created an attenuated strain of Mycobacterium smegmatis containing $M$. tuberculosis genes. The vector conferred better protection against tuberculosis than the standard bacillus Calmette-Guérin (BCG) vaccine in mice. ${ }^{1}$ The not-for-profit Aeras has licensed the technology and is now working with the researchers to determine an optimal combination of genes to use in M. smegmatis.

The only prophylactic for TB is the BCG vaccine, which is prepared from a live, attenuated bacterial strain closely related to M. tuberculosis called M. bovis. In BCG, deletion of the region of difference 1 locus is responsible for its attenuation and loss of virulence. ${ }^{2}$

Although the vaccine protects infants from pulmonary $\mathrm{TB}$, it is not effective at protecting adults, nor can it prevent transmission or clear latent infections.

Albert Einstein College of Medicine's new vaccine originated from research on Mycobacterium secretion systems and their role in helping the bacteria evade the host immune system.

M. tuberculosis employs multiple strategies to do this, including a specialized secretion system encoded by genes in the bacterium's esx-1 locus. The $M$. tuberculosis genome contains four additional es $x$ loci-es $x$ - 2 through es $x$-5-that may encode secretion systems, but their functions are poorly understood.

The researchers were exploring the role of the es $x-3$ locus in immune evasion because it is the only es $x$ locus conserved across all strains of Mycobacterium. ${ }^{3,4}$ The locus is essential for the growth of $M$. tuberculosis and the attenuated M. bovis strain used in BCG. ${ }^{5}$

The team found that unlike M. tuberculosis and BCG, the more distant relative $M$. smegmatis could grow normally when es $x$ - 3 was deleted, and thus they selected the strain to study the locus' role.

In mouse models of $M$. smegmatis infection, an esx-3-deleted strain was unable to evade the host innate immune system, whereas wildtype and esx-1-deleted strains did not elicit a strong innate immune response. Moreover, the es $x$-3-deleted strain had attenuated virulence.

Next, the researchers inserted a set of 26 M. tuberculosis genes including all 11 esx-3 genes into the attenuated M. smegmatis strain. Despite the addition of $e s x-3$ genes, the strain still was not virulent and could not evade the immune system.

At this point, the researchers began to think they had the makings of a vaccine vector in hand.

In proof-of-concept studies in mouse models of lethal M. tuberculosis infection, i.v. or subcutaneous immunization with the recombinant attenuated $M$. smegmatis strain increased survival and resulted in greater decreases in tissue bacterial load compared with immunization using the BCG vaccine. Several of the mice receiving the recombinant $M$. smegmatis vaccine had tissue bacterial load reductions that were over 1,000-fold greater than those for mice given BCG.

Results were published in Nature Medicine.

"This is the first time we have seen a vaccine that can produce over three-log reductions in tissue bacterial burdens over BCG and a bactericidal immune response that causes a sustained decline in bacterial load," said William Jacobs Jr., corresponding author on the paper and a professor in the Department of Microbiology and Immunology and the Department of Genetics at Albert Einstein College of Medicine.

"What we've been seeing with TB vaccine candidates up until now are around one-log reductions in bacterial loads over BCG and immune responses that result in growth inhibition," he added.

Jacobs, who also is an investigator at the Howard Hughes Medical Institute, said the recombinant $M$. smegmatis vaccine vector is easy to modify because the genes in the cosmid insert could be swapped out with those encoding antigens from $M$. tuberculosis that are known to elicit an adaptive immune response.

Cosmids are plasmid-type DNA vectors that can be used to deliver recombinant genes.

"You may also be able to use this vector to create vaccines for other diseases by using the cosmid to insert genes encoding the antigens that are relevant to those diseases," added Jacobs.

"It is very interesting that they were able to use M. smegmatis as a vector to express TB genes because it is a much more distant relative to TB compared with BCG," said Peter Andersen, a professor of infectious disease and VP of vaccine R\&D at the State Serum Institute (SSI).

He said the mouse data suggest that a vaccine using recombinant $M$. smegmatis could prime the immune system more strongly against M. tuberculosis antigens than BCG.

Thomas Evans, CSO at Aeras, said the organization licensed the technology for three reasons. First, M. smegmatis is fast and easy to grow, which suggests it could potentially be used to create vaccine candidates that would be cheap and easy to manufacture. Second, the organization has been looking to expand its portfolio of vaccine candidates to avoid having multiple "me too" approaches in development. Finally, Evans said Aeras is interested in vaccination strategies that can circumvent a pathogen's ability to evade the immune system. 
Aeras has five clinical-stage TB vaccine candidates in development and expects to have a sixth enter clinical trials by year end. The two most advanced are MVA85A and AERAS-402/Crucell Ad35, both of which are in Phase IIb testing.

MVA85A is a recombinant modified vaccinia virus Ankara expressing $M$. tuberculosis antigen $85 \mathrm{~A}$. The vaccine is being developed with the Oxford-Emergent Tuberculosis Consortium Ltd., a joint venture between Emergent BioSolutions Inc. and the University of Oxford. AERAS-402/Crucell Ad35 is an adenovirus serotype 35 (Ad35) vector that expresses $M$. tuberculosis antigen $85 \mathrm{~B}$, $85 \mathrm{~A}$ and the TB10.4 antigen. It is being developed with Johnson \& Johnson's Crucell N.V. unit.

\section{Building the candidate}

Both Jacobs and Evans noted that several aspects of their bacterial strain need to be optimized before it can be used to create an actual vaccine candidate.

For example, Jacobs said his group is working to improve the stability of the cosmid antigen expression system.

Also, Evans noted that Aeras and the group at Albert Einstein College of Medicine are working together to determine the minimum set of genes to delete from and insert into M. smegmatis to elicit a protective immune response against TB.

"We need to determine the optimal M. smegmatis backbone to use and figure out which exact genes in the es $x$-3 locus to delete," Evans told SciBX. "Likewise, we need to narrow down the set of genes we insert by determining the key set of immunoprotective TB genes."

Andersen noted that the H4 TB subunit vaccine being developed by his group at SSI in partnership with Sanofi includes the TB10.4 antigen from M. tuberculosis, which is encoded by a gene in the esx-3 locus.
He said the researchers also should consider evaluating the potential use of the recombinant $M$. smegmatis in a booster vaccine because the strain could be sufficiently different from BCG and $M$. tuberculosis to be used in such a setting.

Evans estimates that the organization is more than a year away from having a preclinical vaccine candidate that uses the recombinant M. smegmatis vector.

The Albert Einstein College of Medicine of Yeshiva University has a pending patent covering the vaccine platform described in the paper. Aeras has licensed the technology for use in TB. Applications to other indications are still available for licensing.

Lou, K.-J. SciBX 4(37); doi:10.1038/scibx.2011.1033

Published online Sept. 22, 2011

\section{REFERENCES}

1. Sweeney, K.A. et al. Nat. Med.; published online Sept. 4, 2011; doi:10.1038/nm.2420

Contact: William R. Jacobs Jr., Albert Einstein College of Medicine of Yeshiva University, New York, N.Y e-mail: jacobsw@hhmi.org

2. Pym, A.S. et al. Mol. Microbiol. 46, 709-717 (2002)

3. Gey Van Pittius, N.C. et al. Genome Biol. 2, RESEARCH0044 (2001)

4. Stinear, T.P. et al. Genome Res. 18, 729-741 (2008)

5. Siegrist, M.S. et al. Proc. Natl. Acad. Sci. USA 106, 18792-18797 (2009)

\section{COMPANIES AND INSTITUTIONS MENTIONED}

Aeras, Rockville, Md.

Albert Einstein College of Medicine of Yeshiva University, New York, N.Y.

Emergent BioSolutions Inc. (NYSE:EBS), Rockville, Md. Howard Hughes Medical Institute, Chevy Chase, Md. Johnson \& Johnson (NYSE:JNJ), New Brunswick, N.J. Sanofi (Euronext:SAN; NYSE:SNY), Paris, France State Serum Institute, Copenhagen, Denmark University of Oxford, Oxford, U.K. 